\title{
sciendo
}

Transport and Telecommunication, 2020, volume 21, no. 1, 61-68

Transport and Telecommunication Institute, Lomonosova 1, Riga, LV-1019, Latvia

DOI 10.2478/ttj-2020-0005

\section{PERFORMANCE ANALYSIS OF RESOURCE SHARING DURING DOWNLINK MULTI-USER TRANSMISSIONS IN CSMA/ECA FULL DUPLEX WLANS}

\author{
Anastasios C. Politis ${ }^{1}$, Hristos T. Anastassiu ${ }^{2}$ \\ International Hellenic University \\ Department of Information, Computer and Telecommunications Engineering \\ Serres, Greece, Terma Magnisias \\ Ianpol@teicm.gr, ${ }^{2}$ hristosa@teicm.gr
}

\begin{abstract}
Full Duplex (FD) wireless communications is considered to be the next big step for future Wireless Local Area Networks (WLANs). Old (IEEE 802.11ac) and new (IEEE 802.11ax) WLAN features are expected to co-exist with FD operation. Some of these features include Downlink Multi-User (DL MU) transmissions at the physical layer while at the Medium Access Control (MAC) layer techniques such as the Transmission Opportunity (TXOP) sharing mechanism facilitates these multiuser transmissions. The traditional Carrier Sense Multiple Access with Collision Avoidance (CSMA/CA) protocol is inadequate to support full duplex communications efficiently. A very promising adaptation of CSMA/CA and a powerful candidate as the basic channel access method for future WLANs is the Carrier Sense Multiple Access with Enhanced Collision Avoidance (CSMA/ECA). In this paper, we provide an analytical model to assess the performance of resource sharing techniques in full duplex WLANs operating with the CSMA/ECA protocol. Our analysis is based on Markov chains to obtain an estimate of the achievable throughput of the Quality of Service Access Point (QAP) during DL MU transmissions.
\end{abstract}

Keywords: Full Duplex Wireless Local Area Networks, Downlink Multi-User, Transmission Opportunity sharing, Carrier Sense Multiple Access/Enhanced Collision Avoidance

\section{Introduction}

The IEEE 802.11ax amendment (IEEE 802.11ax, 2018) focuses on augmenting the efficiency of WLANs, especially when they operate with large numbers of active Quality of Service (QoS) wireless stations (QSTAs). To establish High Efficiency (HE) in dense user WLANs, various enhancements are under consideration by the IEEE 802.11ax Task Group (TG). Based on its predecessor, the new amendment will include several modifications in the PHY (Physical) layer (Khorov et.al., 2019). The most important feature that is introduced by the IEEE 802.11ax amendment is Orthogonal Frequency Division Multiple Access (OFDMA). OFDMA is new in WLANs but it is not a new technology in wireless communications. With OFDMA the frequency spectrum is divided into slices called Resource Units (RUs). Multiple QSTAs are assigned a different slice of the available spectrum, enabling them to receive or transmit simultaneously. The allocation of RUs to QSTAs is performed centrally by the QAP by transmitting special frames called trigger frames.

Downlink Multi User Multiple Input Multiple Output (DL MU-MIMO) was a feature firstly introduced in the IEEE 802.11ac amendment (IEEE 802.11ac, 2013) and is also supported by IEEE 802.11ax. In IEEE 802.11ax, DL MU-MIMO enables a QAP to transmit towards a maximum of 8 stations simultaneously via different spatial streams. OFDMA may be combined with MU-MIMO to further increase system efficiency.

Even though OFDMA and MU-MIMO enable simultaneous transmissions, these transmissions are unidirectional. Enabling bidirectional (uplink and downlink) in-band simultaneous transmissions requires Self Interference Cancellation (SIC) techniques. This very interesting feature, commonly known as Simultaneous Transmit-Receive (STR), was initially under consideration by the IEEE 802.11ax TG. STR operation mode essentially enables FD communication free from collisions among uplink and downlink transmissions. However, the support for the STR operation mode was ultimately abandoned by the IEEE 802.11ax TG, as it was considered a feature out of the scope of the task group (Khorov et.al., 2019). The IEEE 802.11 WG assigned the investigation of STR technical feasibility to a Topic Interest Group (TIG) which has already finished its activity by considering it a feasible and highly advantageous technology. Hence, STR is expected to be included in future amendments (beyond IEEE 802.11ax) (Lopez-Perez et. al., 2019). 
Nevertheless, the exciting feature of FD communications must also be supported by a suitable MAC-layer protocol. The standard CSMA/CA channel access method is not a good solution for these networks. A very promising alternative is the CSMA/ECA protocol which is likely to replace CSMA/CA in FD capable WLANs (Bellalta, 2016). CSMA/ECA outperforms CSMA/CA while providing fair coexistance with legacy CSMA/CA stations (Barcelo et. al., 2009). Prototype hardware implementations already exist (Sanabria-Russo et. al., 2017), and research papers have emerged considering CSMA/ECA as the channel access protocol in full duplex WLANs (Aijaz et. al., 2019), (Salehi et. al., 2019).

Future WLANs (IEEE 802.11ax and beyond) will likely include most of the QoS features defined in the previous IEEE 802.11 amendments. One of these features introduced by IEEE 802.11ac is the TXOP sharing protocol which facilitates the DL MU-MIMO transmissions. It is a MAC-layer mechanism aiming at increasing the efficiency of the Enhanced Distributed Channel Access (EDCA) functionality at the QAP. However, the TXOP sharing performance has not been investigated with the CSMA/ECA protocol.

In this paper we provide an analytical model based on Markov chains to obtain a performance estimate of the resource sharing mechanisms during DL MU transmissions in FD-capable WLANs exploiting CSMA/ECA as their access protocol. The performance is measured in terms of throughput achieved by utilizing resource sharing and compared to that without resource sharing. To our knowledge, this is the first time that resource sharing protocols (such as the TXOP sharing mechanism) is evaluated in full duplex WLANs with CSMA/ECA as the medium access mechanism.

The rest of the paper is structured as follows: Section 2 provides some MAC-layer background information related to the standard EDCA, TXOP sharing and CSMA/ECA mechanisms. Section 3 presents our proposed Markov chain model to analyse the TXOP sharing functionality with CSMA/ECA. In Section 4 the throughput results are illustrated and the paper is concluded in Section 5 with final remarks.

\section{Background}

\subsection{EDCA and TXOP Sharing}

According to EDCA (IEEE 802.11e, 2005), a wireless node (QAP and QSTA) will implement four priority queues known as Access Categories (ACs). Each $\mathrm{AC}$ is assigned to a different traffic class: $A C[V O], A C[V I], A C[B E]$ and $A C[B K]$ serve voice (VO), video (VI), best effort (BE) and background $(\mathrm{BK})$ traffic, respectively. The priorities for each $\mathrm{AC}$ are assigned in descending order, with $A C[V O]$ owning the highest priority and $A C[B K]$ having the smallest probability of channel access.

Each AC acts as an individual station inside a wireless node. Hence, an AC of a wireless station contents for channel access among the remaining ACs owned by that node (intra-AC contention) and among the ACs of other nodes in the WLAN (inter-AC contention). Each AC will perform an instance of the standard CSMA/CA channel access protocol.

In an attempt to increase efficiency, the IEEE 802.11e amendment defines that once an AC acquires channel ownership it may transmit sequentially multiple frames in the form of a Contention-Free Burst (CFB), for a specific period. The time that is available for the CFB is known as TXOP limit. The CFB feature is available only to the multimedia ACs (i.e., $A C[V O]$, and $A C[V I]$,). Non-multimedia ACs are allowed one frame transmission per channel access. The IEEE 802.11ac amendment extended the TXOP resource reservation mechanism due to the DL MU-MIMO capability enabled at the QAP. This enhanced version is known as TXOP sharing and defines the mode of sharing the CFB period among multiple ACs within a wireless node. An external and internal contention winning AC (primary AC) may allow the remainder ACs (secondary ACs) to transmit their frames simultaneously. If the TXOP is finally shared, it becomes an MU-TXOP.

\subsection{CSMA/ECA and Fair Share}

CSMA/ECA introduces minimal modifications to the legacy CSMA/CA protocol that can lead to a collision-free medium access after a convergence period. To achieve this, the protocol adopts a reservation-based approach similar to Time Division Multiple Access (TDMA) and combines it with the standard random access mechanism already used in WLANs. This makes CSMA/ECA especially useful in WLANs operating in FD mode.

CSMA/ECA, unlike CSMA/CA, defines a deterministic backoff after a station has transmitted successfully. Otherwise, the collision avoidance mechanism is operational as long as frames collide (i.e., the backoff is doubled for each unsuccessful attempt). The system goes through a transitory operation and 
will eventually stabilize reaching steady state where no collisions occur since every station will have a dedicated slot for its attempt. CSMA/ECA specification defines the usage of a virtual frame, $V$, similarly to the concept of a frame in TDMA. $V$ is a collection of slots with some or all slots dedicated to a transmission attempt of every station in the system.

Ideally, the value of $V$ must be set to the number of active nodes in the WLAN so that each slot in the virtual frame contains a transmission attempt. This will lead to the maximum efficiency of the system. However, to ensure backward compatibility with the presence of legacy nodes, $V$ is set to:

$V=\left\lceil\frac{C W_{\min }}{2}\right\rceil-1$

where $\mathrm{CW}$ is the contention window. After a successful transmission the node sets its backoff deterministically to $B_{d}=V$.

In the case of EDCA, each AC runs and instance of the CSMA/ECA protocol with its own MAC layer parameters. CSMA/ECA modifies the EDCA specification in several ways. More specifically, the Arbitration Inter-Frame Space (AIFS) and TXOP are considered as unfit for the operation of CSMA/ECA. Hence, AIFS is eliminated and TXOP is substituted by the Fair Share (FS) algorithm. FS dictates that at steady-state an AC will transmit $2^{i[A C]}$ frames, instead of the number of frames allowed by the TXOP period, as defined in the standard EDCA functionality. Where, $i$ is the backoff stage the AC is currently at. For more details on the operation and efficiency of CSMA/ECA the interested reader may refer to (Sanabria-Russo et. al., 2018).

To enhance FS performance at the AP during DL MU transmissions, we investigate the sharing of FS in a similar fashion with the TXOP sharing mechanism. The primary AC will transmit $2^{i[A C]}$ frames, and if it decides to share its allocated resources, the secondary ACs will transmit simultaneously during the transmission slot of the primary AC. In this way, collision-free schedules are not disrupted and the throughput achieved by the AP can be increased.

\section{System Model}

In this section we present the resource sharing performance analysis of CSMA/ECA which is based on a Markov chain model. We consider the system functioning at steady-state and in saturation conditions i.e., a collision-free schedule is reached and all ACs at the AP have frames ready for transmission. Our first goal is to determine the transmission probability of each $\mathrm{AC}, \pi[q]$, with $q \in[V O, V I, B E, B K]$, and then provide an estimate of the achieved per AC throughput at the AP with TXOP and FS sharing. The analysis provided is based on previous work by (Bianchi, 2000) and (Yazid et. al., 2014).

Figure 1 depicts the Markov model for the CSMA/ECA steady-state operation with resource sharing at the AP.

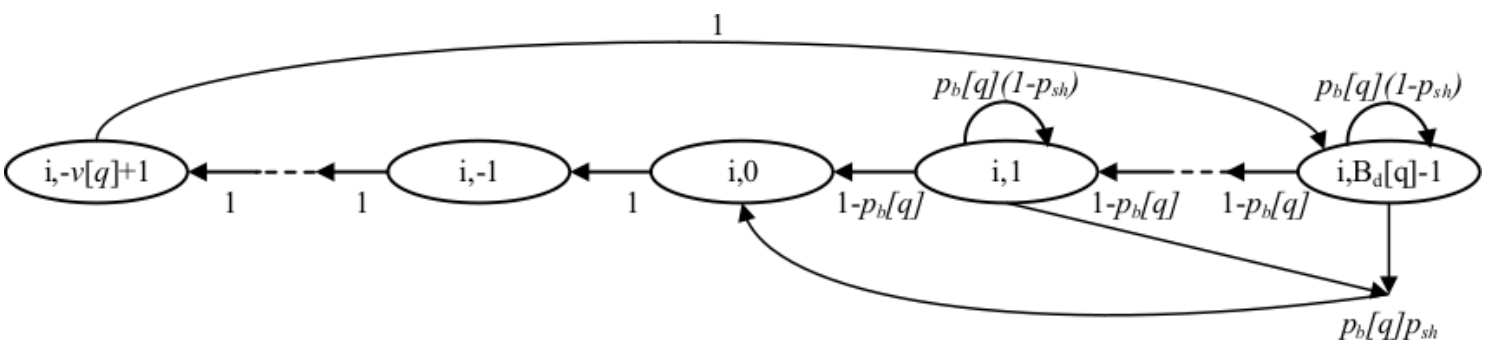

Figure 1. Model of CSMA/ECA during steady-state operation with resource sharing

In the figure, $p_{b}[q]$ represents the blocking probability of an $\mathrm{AC}$, i.e, the probability that an $\mathrm{AC}$ senses the transmission of another AC at the AP. $v[q]$ is the number of frames transmitted by an AC during its transmission slot and is given by:

$v[q]=\left\{\begin{array}{cc}\left\lfloor\frac{T X O P_{l i m}[q]}{T_{M P D U}+T_{B A}+T_{S I F S}}\right\rfloor, & T X O P \\ 2^{i[q],} & F S\end{array}\right.$, 
where, $T X O P_{\text {lim }}[q]$ is the contention-free period of $A C[q] . T_{M P D U}$ and $T_{B A}$ are the transmission times of the data frame and the block acknowledgement (BA) frame, respectively. $T_{S I F S}$ is the short inter-frame space duration. It must be noted that $v[q]=1$ for $q \in[B E, B K]$ according to the TXOP rules of the IEEE 802.11 standard.

An AC at the AP can start a DL MU transmission by using OFDMA, MU-MIMO or both (Khorov et.al., 2019). Hence, the resource sharing probability, $p_{s h}$, can be estimated as $p_{s h}=p_{s h}^{M}+p_{s h}^{O}-p_{s h}^{M} p_{s h}^{O}$, where $p_{s h}^{M}$ is the sharing probability due to the MIMO operation and $p_{s h}^{O}$ due to OFDMA. The former can be defined as $p_{s h}^{M}=A_{u} /_{A_{a}}$, and the latter as $p_{s h}^{o}=R_{u} /_{R_{a}} \cdot A_{u}$ and $A_{a}$ are the number of antennas used by the primary $\mathrm{AC}$ and the total number of antennas available at the AP, respectively. Similarly, $R_{u}$ represents the number of resource units (RUs) used by the primary AC and $R_{a}$ the available RUs in the frequency band. For simplicity, we assume that $p_{s h}$ is the same for all ACs.

Let, $b(t)$ denote the stochastic process representing either the backoff counter $k$, with $k \in\left[0,1, \ldots, B_{d}[q]-1\right]$, or the $k^{\text {th }}$ transmitted frame, with $k \in[1,2, \ldots, v[q]]$, at backoff stage $i$. The stationary distribution of the Markov chain depicted in Figure 1 is:

$b_{i, k}=\lim _{t \rightarrow \infty} P\left\{i, b_{[q]}(t)\right\}$,

with $k \in\left[-v[q]+1, B_{d}[q]-1\right]$. The transition probabilities of the Markov chain are:

$\begin{cases}P\{i, k \mid i, k+1\}=1-p_{b}[q], & k \in\left[0, B_{d}[q]-2\right] \\ P\{i, k \mid i, k\}=p_{b}[q]\left(1-p_{s h}\right), & k \in\left[0, B_{d}[q]-1\right] \\ P\{i, k-1 \mid i, k\}=1, & k \in[0,-v[q]+2] .\end{cases}$

The first equation in (4) represents the fact that an $A C[q]$ decrements its backoff counter when the remaining $\mathrm{AC}$ remain silent. The second equation implies that the backoff countdown is postponed when an $A C[q]$ senses at least another AC transmitting without resource sharing. Lastly, the third equation describes the fact that once the $A C[q]$ reaches its transmission state, multiple frames from that AC may be transmitted sequentially. The number of those frames is bounded by $v[q]$ obtained by (2).

Due to the chain regularities:

$\left\{\begin{array}{c}b_{i, k}=b_{i, 0}, k \in[-1,-v[q]+1] \\ b_{i, k}=\frac{b_{i, k+1}}{-a}, k \in\left[0, B_{d}[q]-2\right] \\ b_{i, k}=\frac{b_{i, 0}}{-a\left(1-p_{b}[q]\right)}, k \in[0,-v[q]+2]\end{array}\right.$

with:

$a=-\frac{1-p_{b}[q]+p_{b}[q] p_{s h}}{1-p_{b}[q]}$.

Owing to difference equations theory, the solution for the Markov chain is:

$b_{i, k}=\frac{b_{i, 0}}{(-a)^{B} d^{[q]-k}\left(1-p_{b}[q]\right)}, k \in\left[1, B_{d}[q]-1\right]$.

By imposing the normalization condition:

$1=\sum_{k=0}^{B_{d}[q]-1} b_{i, k}+\sum_{k=1}^{v[q]-1} b_{i,-k}=b_{i, 0}+\frac{b_{i, 0}}{1-p_{b}[q]} \sum_{k=1}^{B_{d}[q]-1}(-a)^{k-B_{d}[q]}+b_{i, 0}(v[q]-1)$.

Consider that due to geometric series:

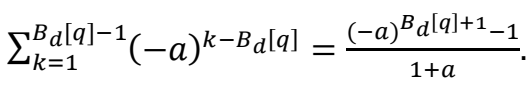

Hence, $b_{i, 0}$ is expressed as:

$b_{i, 0}=\frac{\lambda}{\lambda+(-a)^{-B} d^{[q]+1}-1+\lambda(\nu[q]-1)}$

with:

$\lambda=\left(1-p_{b}[q]\right)(1+a)$.

Note that (10) holds for $p_{s h}>0$. 
Now, the transmission probability, $\pi[q]$, of an AC at the AP can be obtained as the sum of all probabilities of transmission states:

$\pi[q]=\sum_{k=0}^{v[q]-1} b_{i-k}=\frac{v[q] \lambda}{\lambda+(-a)^{-B} d^{[q]+1}-1+\lambda(v[q]-1)}$.

The deterministic backoff countdown of an AC is blocked when at least one of the other ACs at the AP transmits:

$p_{b}[q]=1-\prod_{h \neq q}(1-\pi[h])$.

Equations (12) and (13) can be solved by means numerical methods. Considering all ACs active and equally-sized data frames of length $l_{M P D U}$ backlogged at all of them, an estimate of the achieved throughput per AC can be obtained.

First, let us express the average amount of successfully transmitted data of an AC in a transmission slot as below (Yazid et. al., 2014):

$L[q]=\pi[q] v[q] l_{M P D U}+(1-\pi[q]) p_{s h} \times \frac{\sum_{h \neq q} \pi[h] v[h] l_{M P D U}}{\sum_{h \neq q} \pi[h]}$.

The average duration of the transmission slot may be written as:

$\sigma=\frac{\sum_{h=1}^{q} \pi[h] T_{t}[h]}{\sum_{h=1}^{q} \pi[h]}$

with:

$T_{t}[h]=v[h]\left(T_{M P D U}+2 T_{S I F S}+T_{B A}\right)-T_{S I F S}$

being the data transmission time of the specific AC. $T_{S I F S}$ is the duration of the Short Inter-frame Space (SIFS), $T_{M P D U}$ is the transmission time of the MAC-layer Protocol Data Unit (MPDU) and $T_{B A}$ is the transmission duration of the Block Acknowledgment (BA) frame. Note that the AIFS duration is absent from (16) due to the specification of CSMA/ECA (Sanabria-Russo et. al., 2018). Lastly, the throughput of $A C[q]$ can now be computed as:

$S[q]=\frac{L[q]}{\sigma}$.

\subsection{Model adaptation without resource sharing}

In the special case of $p_{s h}=0$, the Markov chain depicted in Figure 1 reduces to the one representing the case where resource sharing is not used (Figure 2).

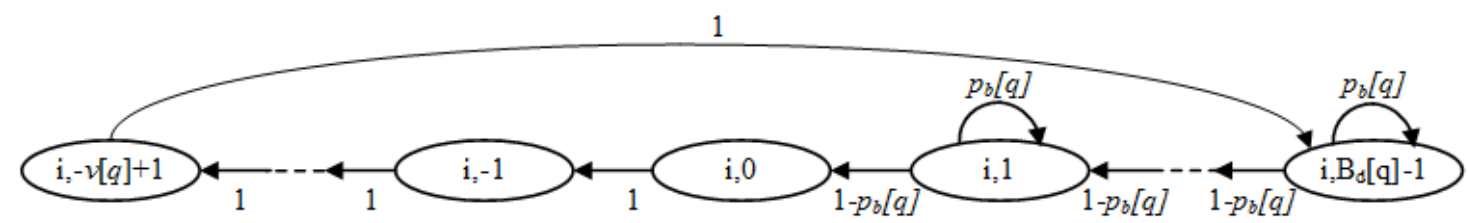

Figure 2. Model of CSMA/ECA during steady-state operation without resource sharing

One can adapt the model presented and easily express the probability of transmission of $A C[q]$. Since $p_{s h}=0, a=-1$ and (7) becomes:

$b_{i, k}=\frac{b_{i, 0}}{\left(1-p_{b}[q]\right)}, k \in\left[1, B_{d}[q]-1\right]$.

Finally, the normalization condition produces:

$\pi[q]=\frac{v[q]\left(1-p_{b}[q]\right)}{B_{d}[q]-1+v[q]\left(1-p_{b}[q]\right)}$.

The set of (19) and (13) can also be solved by exploiting numerical methods. The obtained $\pi[q]$ can be used to provide an estimate of the throughput of $A C[q]$ without resource sharing. 


\section{Results}

The parameters shown in Table 1 are used to evaluate and compare the performance of the resource sharing mechanisms. In the table, $R$ denotes the rate, Pre is the preamble duration and $H d r$ is the header length. Note that the $i$ values in Table 1 lead to the maximum number of contending nodes with all ACs active in a centention-free schedule (Sanabria-Russo et. al., 2018).

Table 1. PHY amd MAC parameters used for numerical results

\begin{tabular}{|l|l|}
\hline PHY & MAC \\
\hline$T_{S I F S}=16 \mathrm{us}$ & $i[\mathrm{VO}, \mathrm{VI}, B E, B K]=5,4,3,3$ \\
\hline$R($ data $)=1201 \mathrm{Mbps}$ & $\mathrm{Hdr}($ data, control $)=240 \mathrm{bits}$ \\
\hline$R($ control $)=282 \mathrm{Mbps}$ & $C W_{\min }[\mathrm{VO}, \mathrm{VI}, B E, B K]=8,16,32,32$ \\
\hline $\operatorname{Pr} e($ data $)=68.8 \mathrm{us}$ & $T X O P_{\text {lim }}[\mathrm{VO}, \mathrm{VI}, B E, B K=1504,3008 \mathrm{us}$ \\
\hline $\operatorname{Pre}($ data $)=64.8 \mathrm{us}$ & $M P D U$ size $=11454$ bytes \\
\hline
\end{tabular}

By using Matlab we have obtained an estimate for the transmission probability, $\pi[q]$, for all ACs during DL MU transmissions with and without resource sharing. Figure 3 presents those results for TXOP (Figure 3(a)) and FS sharing (Figure 3(b)).

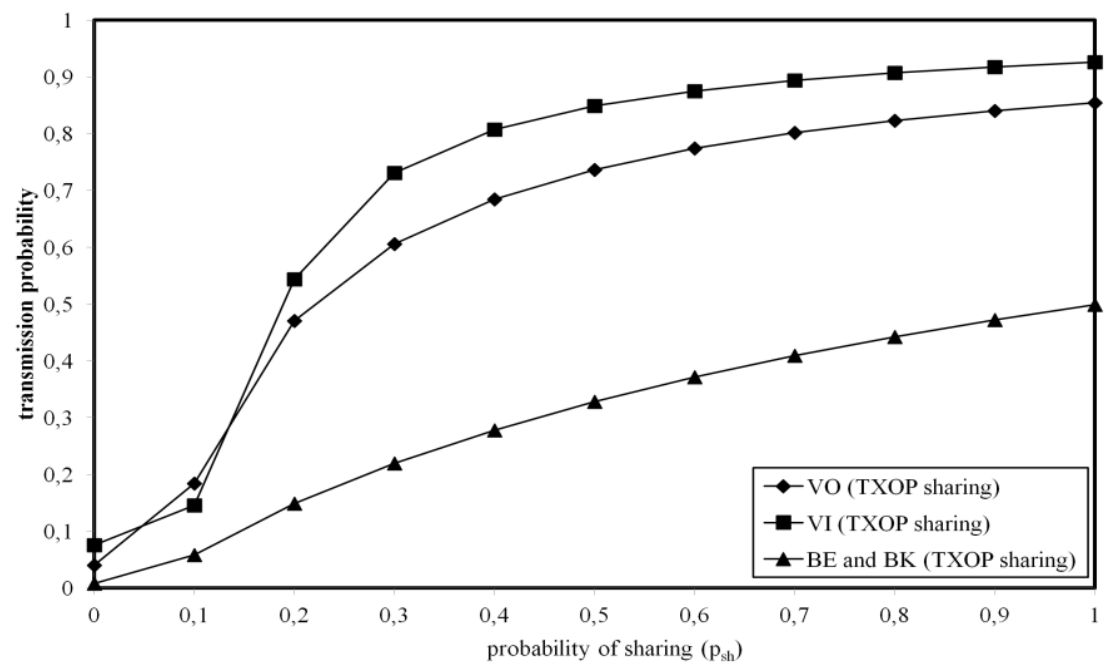

(a)

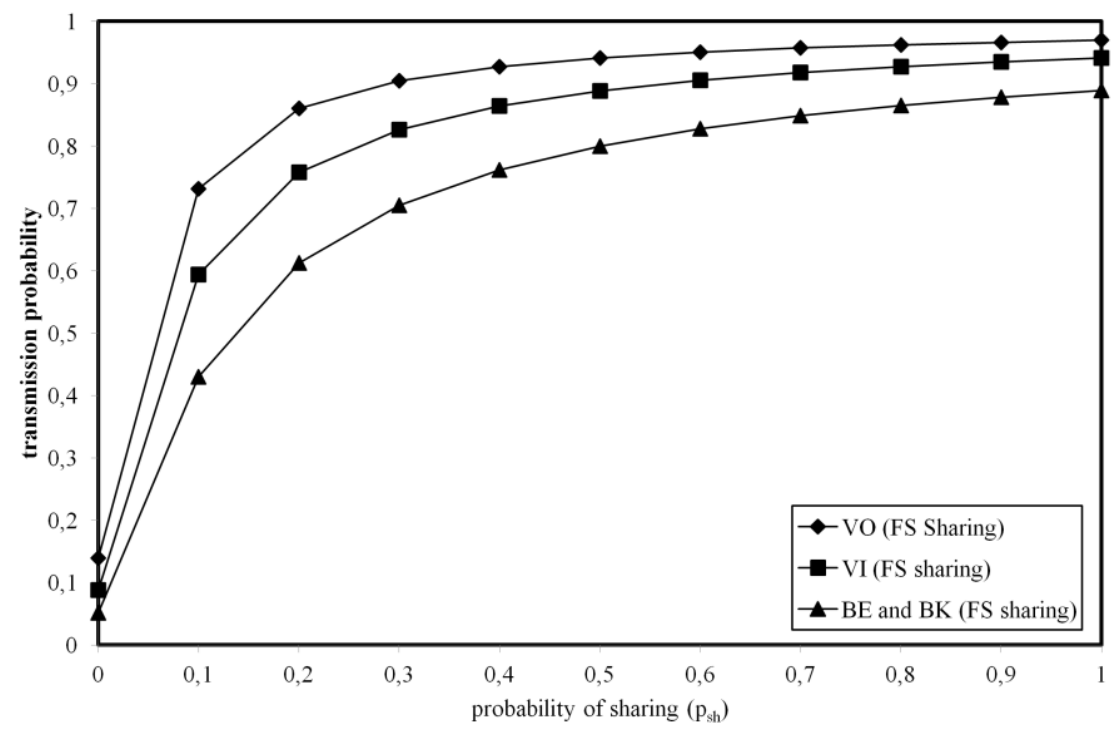

(b)

Figure 3. Per AC throughput with resource sharing according to the probability of sharing (a) TXOP sharing (b) FS sharing 
It is clear that by increasing the sharing probability, the transmission probability of an AC also increases. This is an observation that is expected since as resource sharing becomes more probable, ACs that are at the backoff stage will have an increased chance to access the channel.

Figure 4 depicts the throughput results obtained per AC by applying the resource sharing model presented.

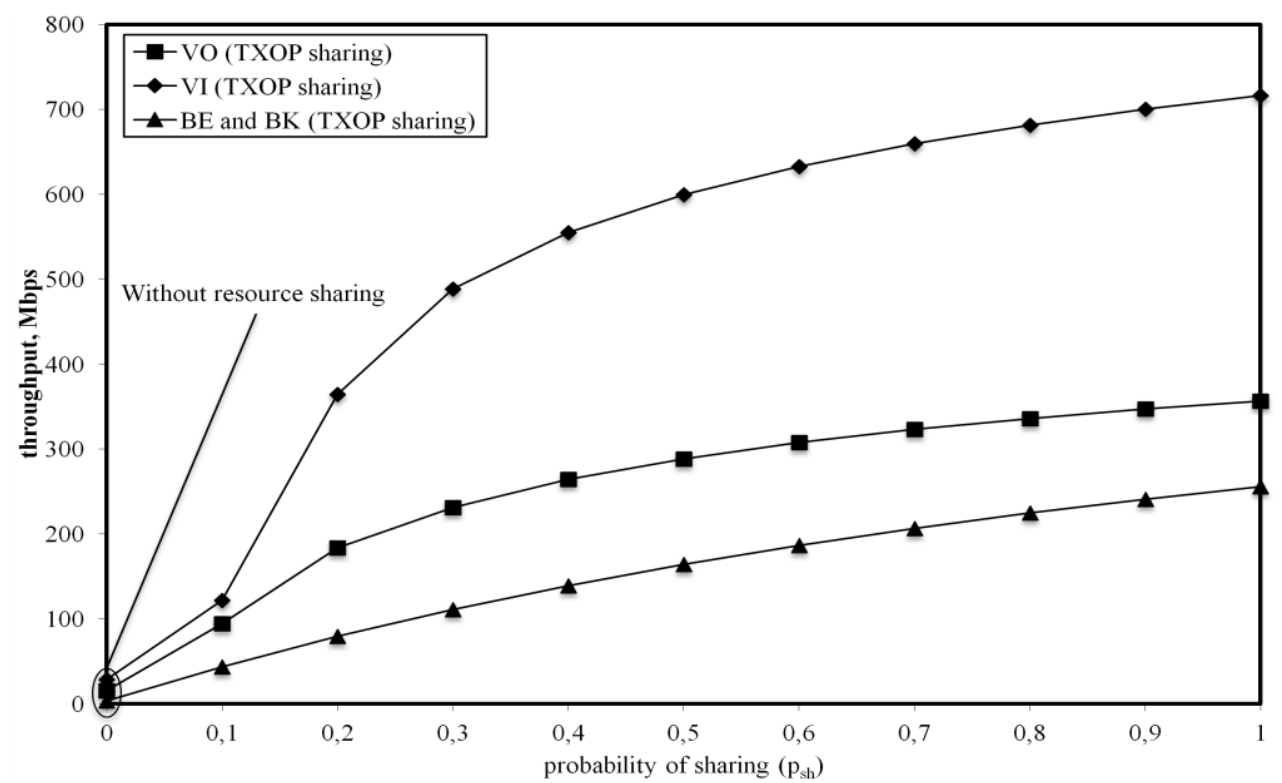

(a)

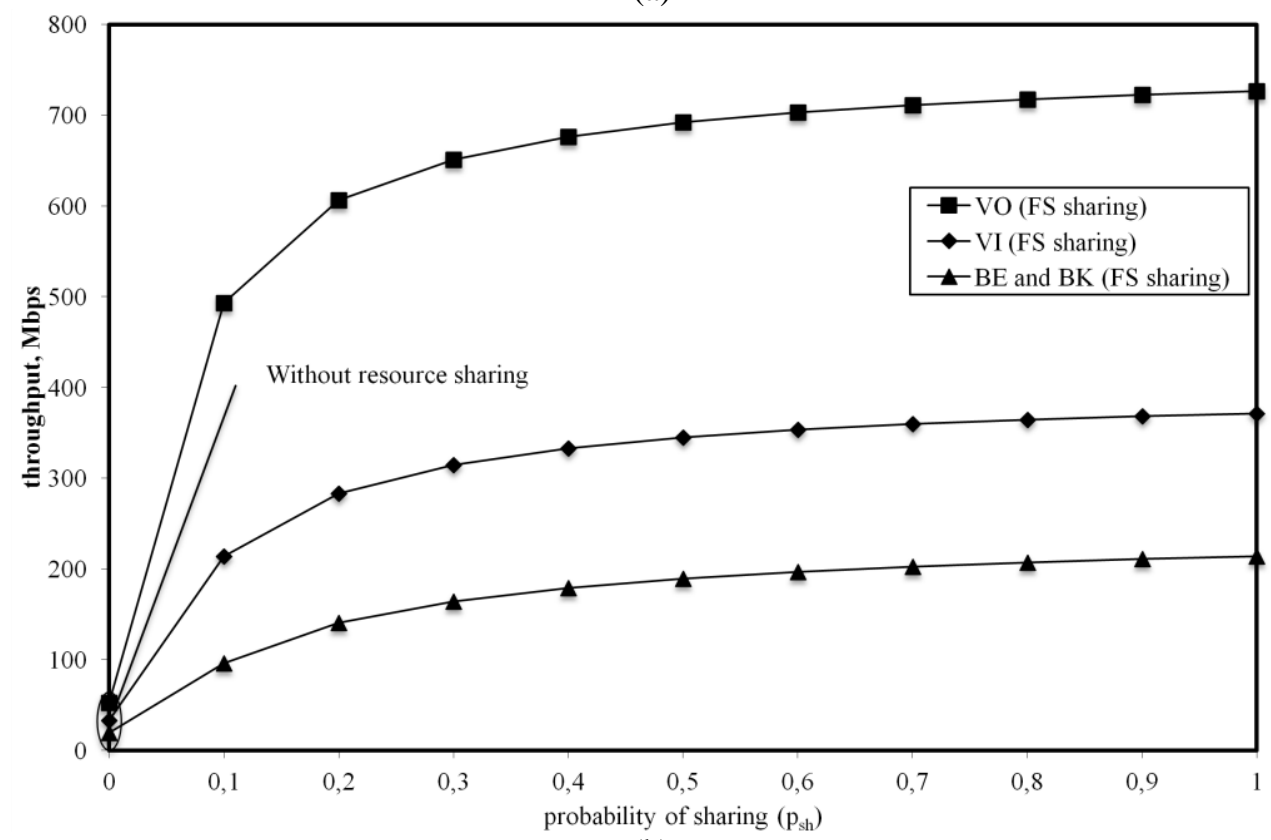

(b)

Figure 4. Per AC throughput with resource sharing according to the probability of sharing (a) TXOP sharing (b) FS sharing

First, note that the BE and BK ACs will perform identically since their values of $v[q], C W_{\min }$ and $i[q]$ are the same. Hence, they will have the same $\pi_{i}[q]$ and exhibit the same throughput. Therefore, their performance is plotted as a common single line.

Secondly, one can observe that the VI AC with TXOP sharing outperforms the VO AC (Figure $3(\mathrm{a}))$. This is also an expected result since the value $v[q]$ obtained by (2) for $A C[V I]$ is more than twice the value of $v[q]$ obtained for $A C[V O]$ (more specifically, $v[V I]=13$ and $v[V O]=6$ ). For FS sharing the opposite is observed (Figure 3(b)), i.e., $A C[V O]$ exhibits a higher throughput performance than $A C[V I]$ since $v[V O]>v[V I]$ in this case. 
From the results obtained in this section, one can conclude that performing resource sharing leads to a significant throughput increment as the probability of sharing increases. Since the OFDMA technology may be combined with MU-MIMO, this probability will become higher in modern WLAN systems. Furthermore, it can be noted that the overall throughput of all ACs achieved with FS sharing is greater than the one obtained with TXOP sharing. Thus extending the FS algorithm with the sharing capability proposed in this paper, will lead to an overall higher throughput performance in multi queue (QoS) FD WLANs.

\section{Conclusions}

We have presented, for the first time, a throughput performance model for resource sharing mechanisms in future full duplex WLANs based on the CSMA/ECA protocol. Results indicate a significant throughput improvement when resource sharing techniques are used. By combining OFDMA and MU-MIMO in the downlink the sharing possibility becomes greater. Thus enhancing CSMA/ECA with resource sharing mechanisms in future FD-capable WLANs will lead to significant performance improvements.

\section{Acknowledgements}

The authors wish to acknowledge financial support provided by the Special Account for Research Funds of the International Hellenic University, Greece, under grant SAT/IE/100419-160/11.

\section{References}

1. IEEE Standard 802.11ax. (2018). Wireless LAN Medium Access Control (MAC) and Physical Layer (PHY) Specifcations Amendment Enhancements for High Efficiency WLAN.

2. Khorov, E., Kiryanov, A., Lyakhov, A., Bianchi, G. (2019) A Tutorial on IEEE 802.11ax high efficiency WLANs, IEEE Communications Surveys and Tutotials, 21(1), 197-215. DOI: 10.1109/COMST.2018.2871099.

3. IEEE Standard 802.11ac. (2013). Wireless LAN Medium Access Control (MAC) and Physical Layer (PHY) Specifications, Amendment 4: Enhancements for Very High Throughput for Operation in Bands below $6 \mathrm{GHz}$.

4. Lopez-Perez, D., Garcia-Rodriguez, A., Galati-Giordano, L., Kasslin, M., Doppler, K. (2019) IEEE 802.11be Extremely High Throughput: The Next Generation of Wi-Fi Technology Beyond 802.11ax. IEEE Communications Magazine, 57(9), 113-119. DOI: 10.1109/MCOM.001.1900338.

5. Bellalta, B. (2016) IEEE 802.11ax: High-Efficiency WLANs. IEEE Wireless Communications, 23(1), 38-46. DOI: 10.1109/MWC.2016.7422404.

6. Barcelo, J., Bellalta, B., Sfairopoulou, A., Cano, C., Oliver, M. (2009) CSMA with Enhanced Collision Avoidance: a Performance Assessment. In: Proceedings of IEEE Vehicular Technology Conference'09, Barcelona, pp. 1-5.

7. Sanabria-Russo, L., Barcelo, J., Bellalta, B. and Gringoli, F. (2017) A High Efficiency MAC Protocol for WLANs: Providing Fairness in Dense Scenarios, IEEE/ACM Transactions on Networking, 25(1), 492-505. DOI: 10.1109/TNET.2016.2587907.

8. Aijaz, A., Kulkarni, P. (2019) Exploiting CSMA/ECA and Adaptive Sensitivity Control for Simultaneous Transmit and Receive in IEEE 802.11WLANs. In: Proceedings of the $25^{\text {th }}$ European Wireless Conference, Aarhus, Denmark.

9. Salehi, S., Li, L., Shen, C-C. and Cimini, L. (2019) Traffic Differentiation in Dense WLANs with CSMA/ECA-DR MAC Protocol. In: Proceedings of the $88^{\text {th }}$ Vehicular Technology Conference, Chicago, IL, USA.

10. IEEE Standard 802.11e. (2005). Wireless LAN Medium Access Control (MAC) and Physical Layer (PHY) Specifications, Amendment 8: Medium Access Control (MAC) Quality of Service Enhancements.

11. Sanabria-Russo, L. and Bellalta, B. (2018) Traffic differentiation in dense collision-free WLANs using CSMA-ECA, Ad hoc Networks, 75-76, 33-51, 2018. DOI: 10.1016/j.adhoc.2018.03.006.

12. Bianchi, G. (2000) Performance analysis of the IEEE 802.11 distributed coordination function, IEEE Journal on Selected Areas in Communications, 18(3), 535-547. DOI: 10.1109/49.840210.

13. Yazid, M., Ksentini, A., Bouallouche-Medjkoune, L., Aissani, D. (2014) Performance analysis of the TXOP sharing mechanism in the VHT IEEE 802.11ac WLANs, IEEE Communications Letters, 18(9), 1599-1602. DOI: 10.1109/LCOMM.2014.2337253. 